\title{
Urban structure analysis of mega city Mexico City using multi-sensoral remote sensing data
}

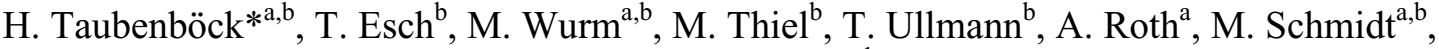 \\ H. Mehl ${ }^{\mathrm{a}}, \mathrm{S}$. Dech $^{\mathrm{a}, \mathrm{b}}$ \\ ${ }^{a}$ German Remote Sensing Data Center (DFD), German Aerospace Center (DLR), Oberpfaffenhofen, \\ 82234 Wessling, Germany \\ ${ }^{\mathrm{b}}$ University of Würzburg, Institute of Geography, Am Hubland, 97074 Würzburg, Germany
}

\begin{abstract}
Mega city Mexico City is ranked the third largest urban agglomeration to date around the globe. The large extension as well as dynamic urban transformation and sprawl processes lead to a lack of up-to-date and area-wide data and information to measure, monitor, and understand the urban situation. This paper focuses on the capabilities of multisensoral remotely sensed data to provide a broad range of products derived from one scientific field - remote sensing to support urban managing and planning. Therefore optical data sets from the Landsat and Quickbird sensors as well as radar data from the Shuttle Radar Topography Mission (SRTM) and the TerraSAR-X sensor are utilised. Using the multi-sensoral data sets the analysis are scale-dependent. On the one hand change detection on city level utilising the derived urban footprints enables to monitor and to assess spatiotemporal urban transformation, areal dimension of urban sprawl, its direction, and the built-up density distribution over time. On the other hand, structural characteristics of an urban landscape - the alignment and types of buildings, streets and open spaces - provide insight in the very detailed physical pattern of urban morphology on higher scale. The results show high accuracies of the derived multi-scale products. The multi-scale analysis allows quantifying urban processes and thus leading to an assessment and interpretation of urban trends.
\end{abstract}

Keywords: Mega city, Urban structure analysis, Urban Remote Sensing, Planning

\section{INTRODUCTION}

The term "urban structure" is ambiguous, comprehensive and abstract. First of all urban structure is defined by the heterogeneous physical alignment and characteristics of buildings, streets and open spaces. In addition, the urban structure can be understood at different levels of spatial resolution. Furthermore it must be understood historically since the elements of which it is comprised undergo continuous transformation and replacement (Moudon, 1997). Apart from the physical structure the spatial pattern of different urban functions, spatial distributions of economic capabilities or social classes of its population characterise and shape urban structure. Thus, the city, seen as a human product, is the physical and architectonic reflection of the society that created it (Gonzalez \& Medina, 2004). On the whole, the dynamic processes of urban transformation and expansion are a complicated social/economic phenomenon linked with details related to topography, transportation, land use, social structure and economic type, but it is generally related to demography and economy in a city (Li et al., 2002). Against this background "urban structure" must be defined as a multilayered term including the temporal dimension of urban transformation, the physical sphere of its objects and the condition of demographic, socio-economic and political influences.

With this in mind this paper focuses on the capabilities of multi-sensoral remote sensing data and methodologies to provide insights to the physical urban structure and its developing over time on one of the largest urban agglomerations on this planet: Mexico City, Mexico. Utilising multi-sensoral remote sensing data the aim is to produce area-wide, multitemporal and up-to-date datasets at different scales to analyse spatial characteristics of urban development and transformation.

*hannes.taubenboeck@dlr.de; phone +49 (0)8153 -28-2480; fax +49 (0)8153-28-1445; www.dlr.de

Remote Sensing for Environmental Monitoring, GIS Applications, and Geology VIII,

edited by Ulrich Michel, Daniel L. Civco, Manfred Ehlers, Hermann J. Kaufmann

Proc. of SPIE Vol. 7110, 71100E - C 2008 SPIE · CCC code: 0277-786X/08/\$18 · doi: 10.1117/12.800272

Proc. of SPIE Vol. $711071100 \mathrm{E}-1$ 
Therefore on the one hand medium and high resolution optical satellite data (Landsat and Quickbird) are utilised: Landsat satellites, available since 1972, enable on a geometric resolution ranging from 79-14.5 m a temporal analysis of urban development. Quickbird data feature a geometric quality of $61 \mathrm{~cm}$ panchromatic, and 2.44 meter for the 4 available multispectral bands. On the other hand active systems add up to complete the range of data sets supporting the analysis of urban structures. An interferometric Digital Elevation Model (DEM) based on X- and C-band data from the SRTM is used as well as stripmap data from TerraSAR-X. The DEM provides pixel spacing of $25 \mathrm{~m}$ and a height accuracy of about six meters. The stripmap data from TerraSAR-X provide a geometric resolution of 3 meters and the swath of $30 \mathrm{~km}$ enables to cover large parts of mega cities with one scene.

Remote sensing techniques have already shown their value in mapping urban areas at various scales, and as data sources for the analysis of urban land cover change (Donnay et al, 2001; Batty \& Howes 2001; Herold et al., 2002). Recent research has used remotely sensed images to quantitatively describe the spatial structure of urban environments and characterize patterns of urban morphology. With respect to the degree of geometric detail different approaches have been presented. Critical on urban footprint level is the description, analysis, and modelling of urban form and its changes using spatial metrics (O’Neill et al, 1988; Herold et al., 2003). These indices -e. g. landscape shape index, largest patch index, etc. - can be used to objectively quantify the structure and pattern of an urban environment (Luck \& Wu, 2002; Herold et al., 2002; Ji et al., 2006; Seto \& Fragkias, 2005; Taubenböck et al., 2008; Zhang et al., 2004). On higher scale Pesaresi \& Ehrlich (2008) summarise various approaches to analyse urban morphology utilising high resolution satellite data. Examples are texture analysis (Pesaresi, 2000; Puissant et al., 2005) or morphological profiles (Pesaresi \& Benediktson, 2001). A different approach utilises the land cover classification with a subsequent urban morphology analysis. Physical parameters like built-up density (Taubenböck et al., 2006), building height or sizes, or roof types (Taubenböck, 2008) are utilised to define homogeneous structure zones on house/block level.

We aimed to address several specific questions on the physical urban structure of mega city Mexico City utilising multisensoral remote sensing data sets:

- What can very different remotely sensed data sets contribute to quantify and describe structural characteristics of urban morphology and what can be reasoned from it?

- Does spatial urbanisation correspond to the demographic development of the mega city?

- How can urban pattern be characterized in dependency of the scale utilising remote sensing data?

\section{TEST SITE MEXICO CITY}

'Mega cities' are now conventionally defined to be large urban agglomerations of 10 million or more (Aguilar, 1999; UN, 2003; Mertins, 1992). The UN (UN, 2007) rank the metropolitan area of Mexico City, Mexico with 19 million inhabitants in 2007 as the third most populous urban area in the world. The recent development of Mexico City has been characterised - in contrary to the prospects made in the past (UNDIESA, 1991) - by a slower demographic growth and a relocation of industrial activities at regional level. This is reflected in the case of Mexico City, where about 18 million inhabitants registered in 2000 are far from the projected population of 25-27 million people by the end of the last century (Figure 1). This seems to point to a real shift in the pattern of urban settlement. It has led to a rapid growth of nearby cities - Toluca, Puebla, Cuernavaca, Queretaro, and Pachuca - constituting an entire polynuclear megalopolis urban pattern as the basic structure of a new urban regional order (Aguilar, 1999; Garza, 2002).

Urban growth is the absolute increase in the physical size and total population of urban areas (Potter, 1992). It is the sum of three processes: net urban migration (in-migration minus out migration), plus urban natural increase (birth minus death), plus geographical extension that transforms the formerly rural zones located in the periphery (or peri-urban parts) of large cities into urban areas (Jacquemin, 1999; Brockerhoff, 2000). While the spatial distribution of poverty in urban areas in Mexico is varied, new settlements that tend to grow in the peri-urban hinterland of cities have a high incidence 
of poverty (Hiernaux, 1999; Ávila, 2001). The pattern of urbanisation of the city is dispersed and chaotic due to the absorption of many rural areas. This expansion has had high social and environmental costs which increase social inequalities and the uneven provision of infrastructure, services, and urban equipment (DRMP, 2006).

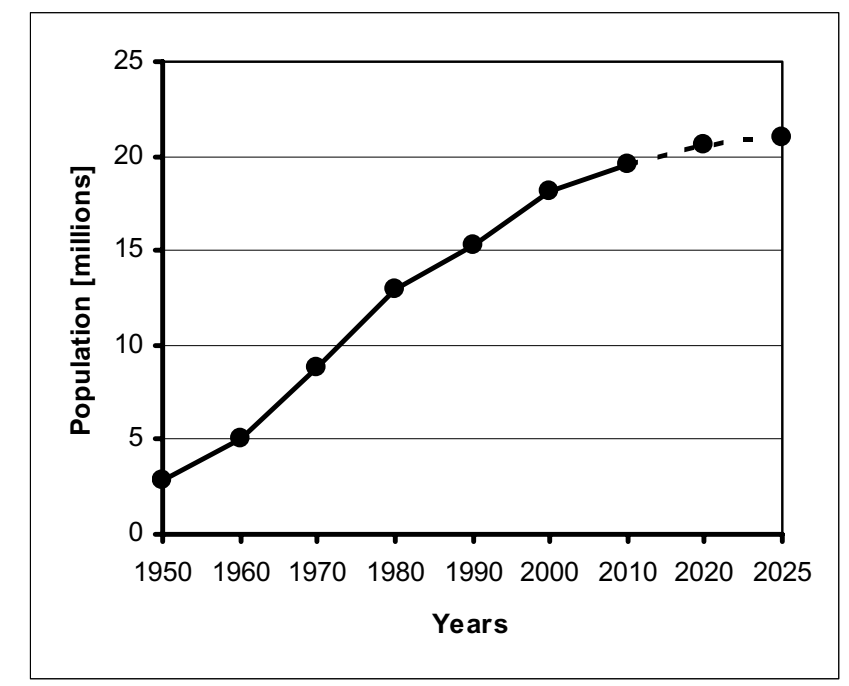

Fig 1. Population development in Mexico, City since 1950 and prospects (UN, 2005)

Mexico City and its metropolitan area are located in the Valley of Mexico (Anáhuac) that lies at an average of 2,240 $\mathrm{m}$ above sea level. The earthquake prone area is surrounded by mountains on all four sides (amongst others the active volcanoes of Popocatepetl and Itzacehuatl), with only one small opening at the north. Thus, Mexico City combines natural hazards with enormous structural vulnerability due to explosive urban sprawl, population density, poverty, etc. Figure 2 shows a perspective view from south-west direction on the valley based on a digital elevation model from SRTM. The overlapping of Landsat data displays the location of the urban footprint of Mexico City embedded in the orography of the landscape, the large extension of urbanised areas, and its urban sprawl into mountainous regions.

\section{METHODOLOGY}

In the following the urban structure analysis of mega city Mexico City is two-fold based on different degrees of detail: On the one hand physical urban sprawl over time is analysed on city level generalising the heterogeneous and small-scale urban landscape. On the other hand utilising high resolution satellite data urban structure is broken down into physical parameters on house/block level to give a detailed perspective on physical characteristics of the urban environment.

\subsection{Medium-resolution change detection utilising multi-sensoral remote sensing data}

The main goal is to identify the urban built-up areas to measure the changes of the urban extension over the time interval. For that purpose the classification methodology is based on an object-oriented hierarchical top-down approach for the time series of Landsat data (Taubenböck, 2008; Taubenböck et al., 2008). The approach utilises spectral, shape and texture features as well as principal component analysis to extract sealed areas from the Landsat data sets. The results are land-cover classifications for the individual available Landsat data sets differentiating urbanised and non-urbanised areas. 


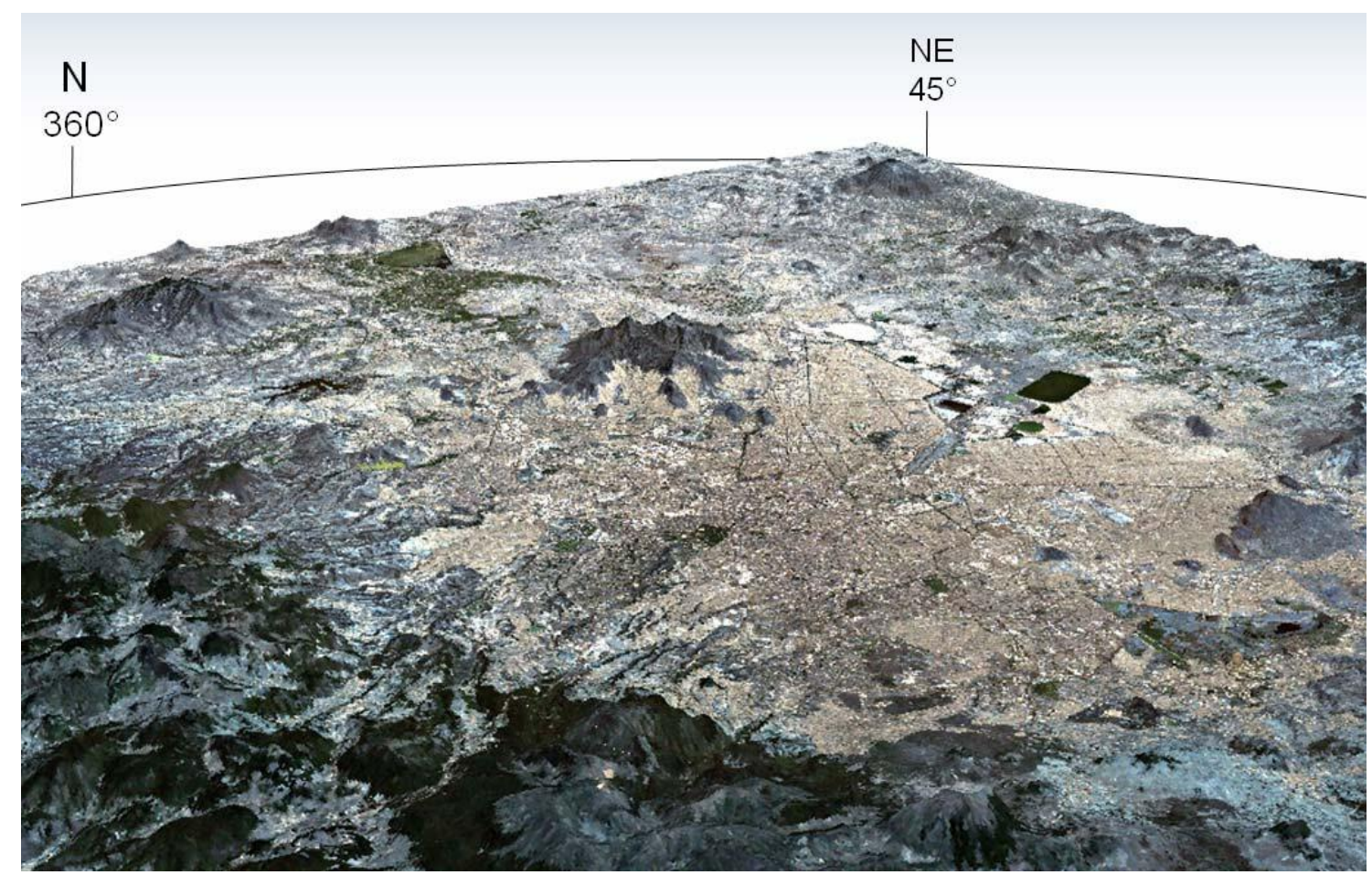

Fig. 2: Overlapping of the DEM (derived from SRTM) with Landsat ETM+ data from 2001 for the megacity Mexico City (Band combinations 3,2,1)

Post classification comparison was found to be the most accurate procedure and presented the advantage of indicating the nature of the changes (Mas, 1999). A comparative analysis of land cover classifications for the available times performed independently was therefore implemented to monitor and analyse the land cover changes in the metropolitan area of Mexico City. Pixelwise change detection was implemented checking the land cover classes individually of the available years. For it, all individual land-cover classifications are sampled up on the highest available geometric resolution of Landsat ETM. Figure 3 shows the result of the change detection displaying the spatiotemporal physical evolution of urbanised areas for the years 1973, 1989 and 2001. Thus, a first result enables to calculate absolute areal growth or assess directions of urban sprawl.

Physical urban structure and pattern may be laminar or punctual, it may increase the density or it may sprawl, it may be mono- or polycentric. The following urban structure analysis is two fold: Firstly, based on the individual classifications of the three time steps histogram analysis of built-up areas on city level identifies mono- or polycentric growth types and their temporal evolution. Therefore a scan algorithm counts the percentage of built-up areas in comparison to non-builtup areas for every row and every column of the classification results. The result of the scan is displayed as continuous graph over the particular spatial location in $\mathrm{X}$ and $\mathrm{Y}$ direction (Figure 3. Plots $\mathrm{X} \& \mathrm{Y}$ ). The peaks reflect the physical focal points of urbanised areas and thus enable to assess the physical spatial pattern as mono- or polycentric. Figure 3 shows the continuous graphs for the three available time steps for Mexico City.

Secondly, built-up density is a measure to characterise spatial urban pattern and structure. Densities vary substantially from city to city and from the urban center to peripheral areas (Taubenböck et al., 2008). Using artificial concentric rings, the built-up density with respect to location is calculated for various spatial zones. The center is defined as the Zocalo, the central plaza of Mexico City. 10 concentric buffer zones are calculated using a $5 \mathrm{~km}$ radius in each case. The ratio of the circles to the particular urbanised areas results in the location-based built-up density. Figure 4 shows built-up density for 10 zones at three available time steps, and thus it also indicates redensification or urban sprawl processes. 


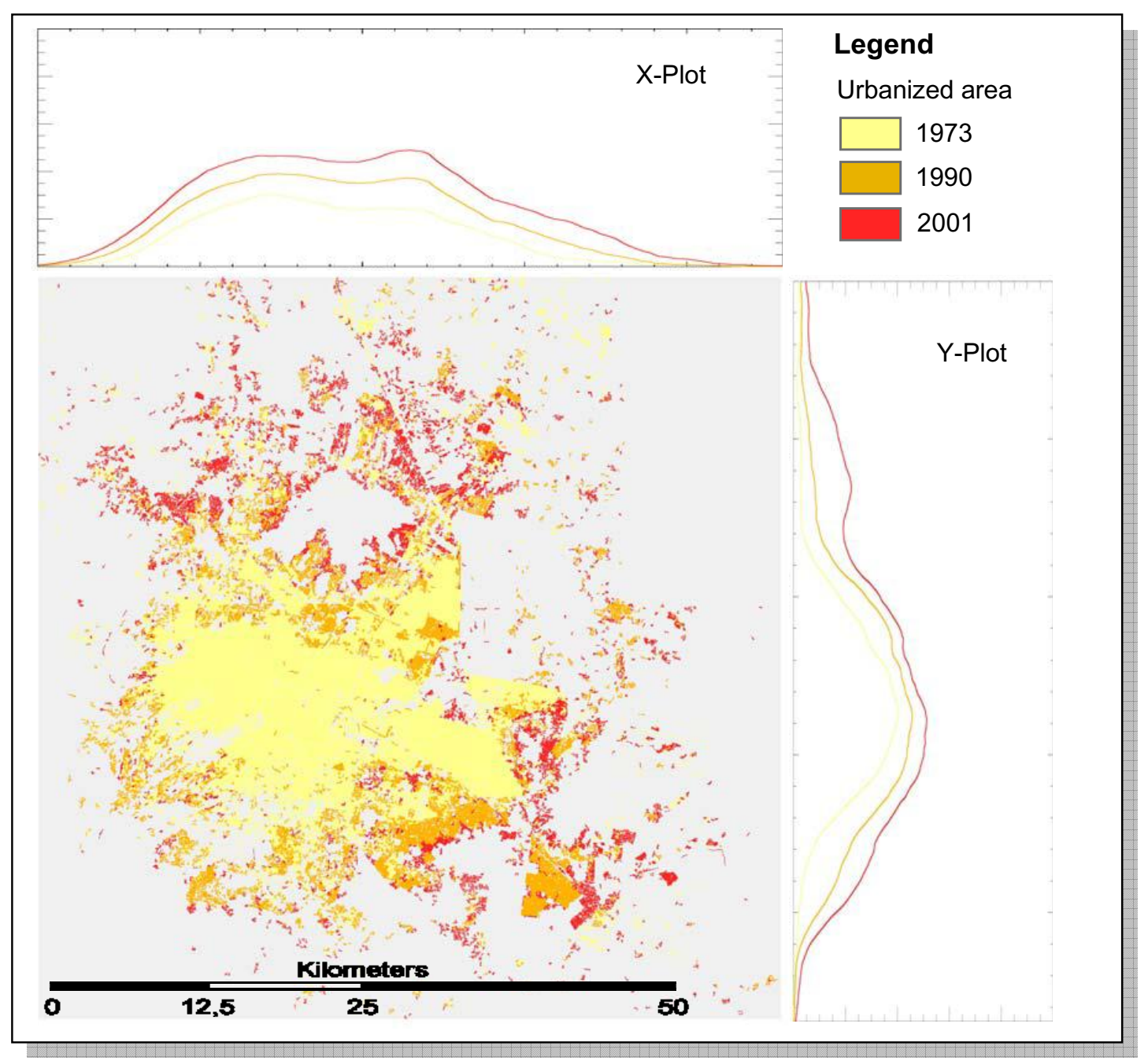

Fig. 3: Change detection of urbanised areas in Mexico City utilising Landsat data from the years 1973, 1990, 2001and histogram analysis

In addition a classification of built-up structures based on stripmap data from TerraSAR-X enables to continue the monitoring of urbanised areas until today. An object-oriented methodology to extract sealed areas has been presented (Breunig, 2008; Esch, 2006; Thiel et al., 2008a; Thiel et al., 2008b). Detection of settlement areas from TerraSAR-X stripmap data was done by the use of speckle divergence and intensity data. Using the optimized segmentation algorithm an object-oriented methodology is utilised to infer built-up areas. By the use of selected features (i. e. shape parameters) a fast and robust rule base was developed and applied to our database. The results show an overall accuracy of nearby 90 $\%$. Figure 5 shows a detail of the TSX-scene of Mexico City (2008) with structural differences appearing in the data. The figure also presents the classification of sealed areas and a first integration of the TSX-classification for postclassification change detection with the Landsat results. 


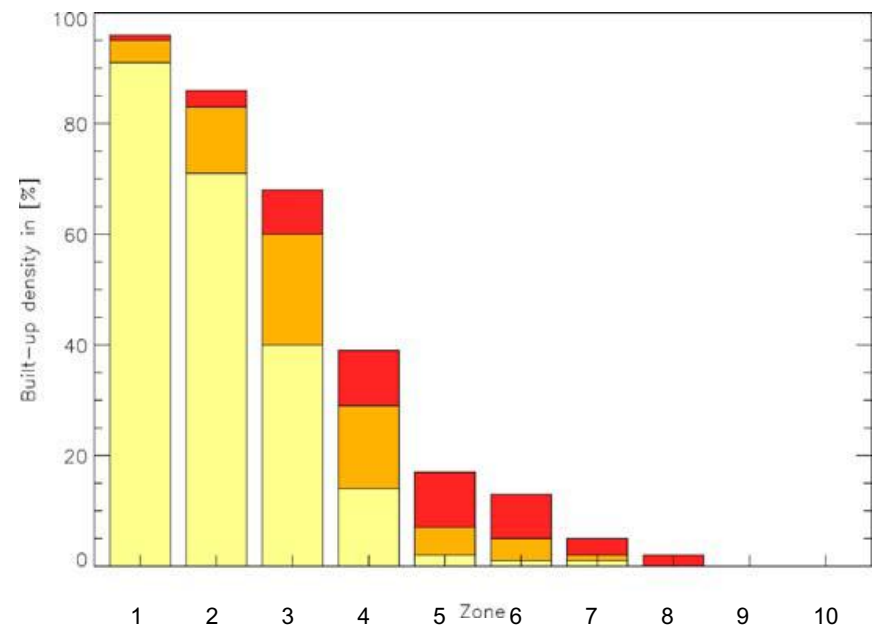

Fig. 4: Location-based built-up-density distribution with increasing distance to the urban core

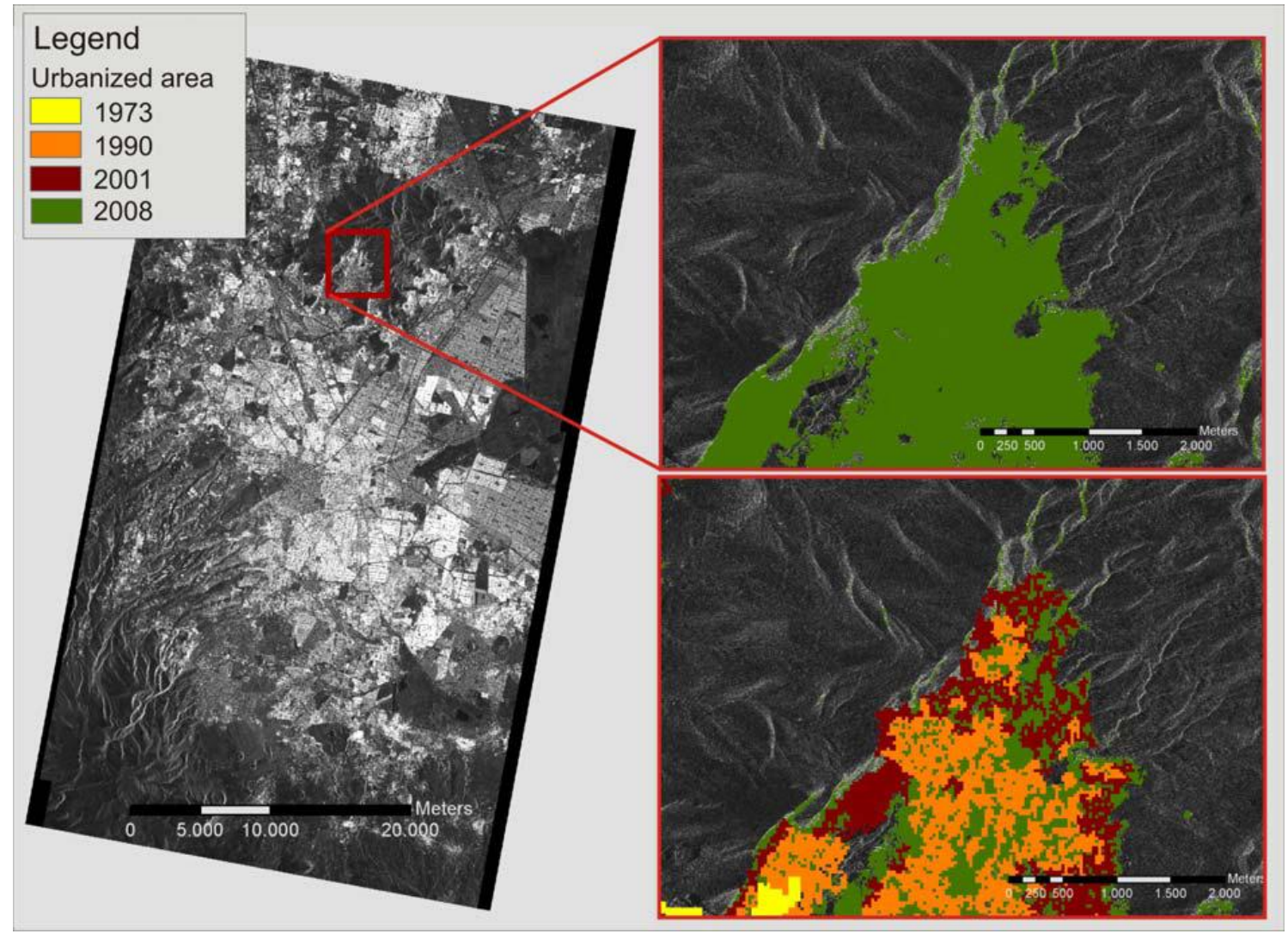

Figure 5: TerraSAR-X data of Mexico City, classification of sealed areas and multisensoral post-classification change detection 


\subsection{High resolution urban structure analysis}

To expand the scale of urban research the use of high resolution optical data (Quickbird) enables to analyse the heterogeneous and small-scale urban morphology on house/block level. The classification methodology is based on an object-oriented hierarchical approach. The object-oriented fuzzy-based methodology was used to combine spectral features with shape, neighbourhood, context and texture features for classification (Taubenböck et al., 2007; Taubenböck 2008). The approach developed on Ikonos data has been transferred to the similar spectral characteristics of Quickbird data of Mexico City. Figure 6b shows the final urban land cover classification. The land-cover classes "buildings", "streets", "vegetation", "shadows", "water", and "bare soil" have been detected. It becomes apparent that single houses were classified as well as the main street network providing a higher geometric as well as thematic detail compared to Landsat data. The urban landscape shows a small-scale structure affected by the alignment of various objects and open spaces. Thus, the built-up density distribution - one example to measure physical urban structure - is spatially highly variable and does not follow any theoretical rules.
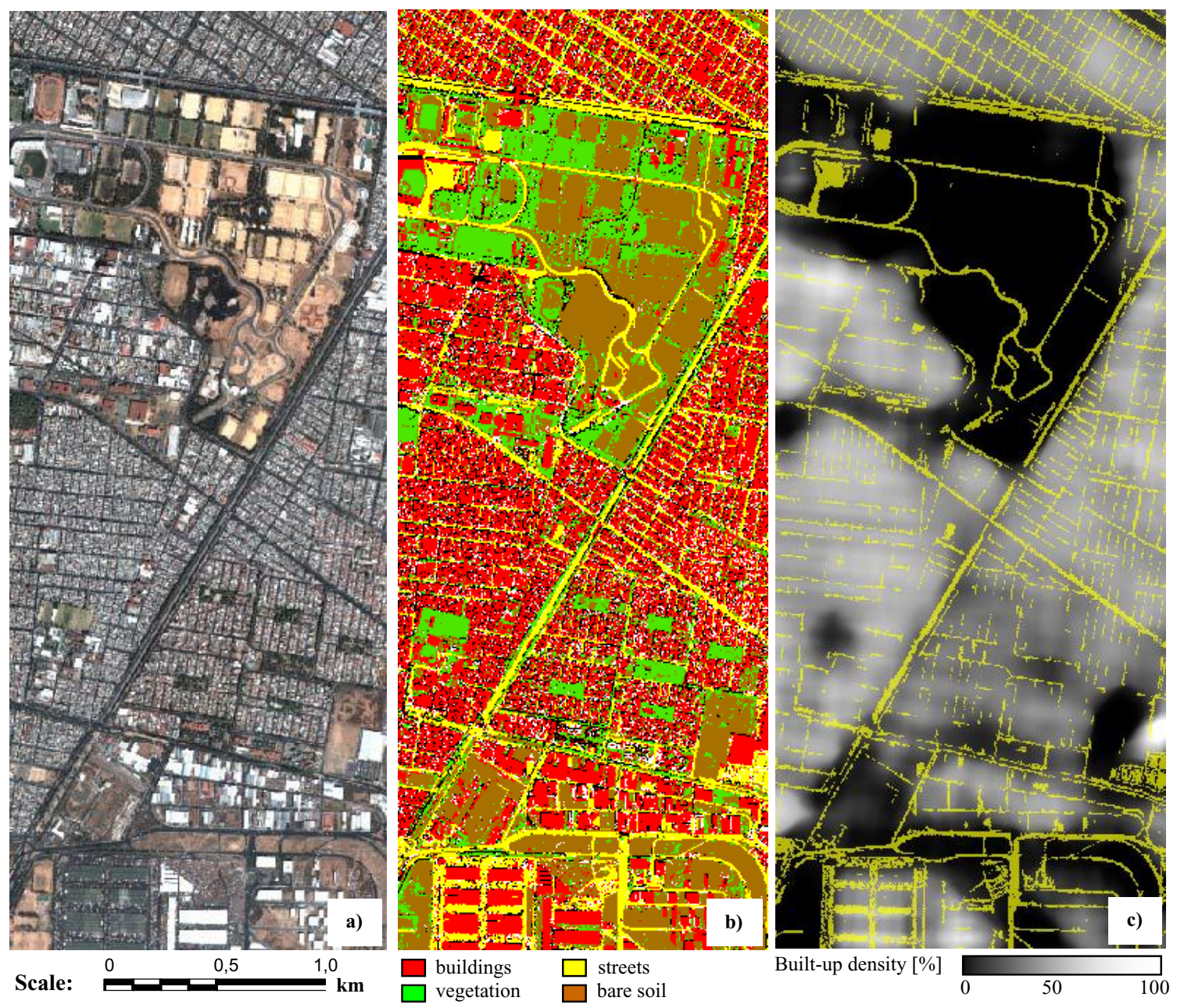

Figure. 6: High resolution QuickBird data (a), land cover classification (b) and built-up density (c) 
Changeovers are calculated based on the data themselves and not on predetermined borders like the street network. A moving window approach scanning the neighbourhood of each house pixel has been implemented. Using this highly variable density distribution Savitzky-Golay filtering fits a polynomial to the data surrounding each data point (density value). The derived polynomial function is utilised to detect built-up density borders. Using a curve-sketching approach the surrounding density values of each point in the urban landscape are analysed. The changeovers are reflected in inflexion points (Taubenböck et al., 2006). The spatial built-up density distribution is shown in figure 6c, characterizing the urban morphologic structure of a central section of the megacity Mexico City. Examples for additional physical parameters characterising the urban morphology are the building sizes, the building heights, the roof types or the building types.

\section{RESULTS AND DISCUSSION}

The accuracy of classifications on medium resolution level is limited due to coarse geometric resolution of the Landsat data. Therefore many "mixed-pixels" containing information on various thematic classes occur. An accuracy assessment has been performed by a randomization of 150 checkpoints per class and a visual verification process for every available data set. The most commonly used method to representing the degree of accuracy of a classification is to build a $\mathrm{k} x \mathrm{k}$ confusion matrix (or error matrix) providing user and producer accuracy as well as the overall accuracy. In addition the Kappa Coefficient has been calculated, a statistical measure of the agreement between two raters who each classify $N$ items into $C$ mutually exclusive categories. It is generally thought to be a more robust measure than simple percent agreement calculation. Furthermore the classification result of the high resolution Quickbird data has been analysed by a visible verification of 150 sample pixels per class. Table 1 presents the accuracies for the particular data set of this multisensoral approach.

Table 1: Accuracy assessment of land-cover classifications derived from multi-sensoral data sets

\begin{tabular}{|c|c|c|c|c|}
\hline Data type & \multicolumn{3}{|c|}{ Landsat data } & TerraSAR-X \\
\hline Time & 1973 & 1989 & 2000 & 2008 \\
\hline Overall accuracy & $88.0 \%$ & $96.0 \%$ & $94.0 \%$ & $91.0 \%$ \\
\hline Kappa & 0.76 & 0.93 & 0.92 & - \\
\hline
\end{tabular}

\begin{tabular}{|l|c|c|}
\hline Data type & \multicolumn{2}{|c|}{ Quickbird data } \\
\hline Classes & User Acc. [\%] & Prod. Acc[\%] \\
\hline Houses & $82.1 \%$ & $79.4 \%$ \\
\hline Vegetation & $86.2 \%$ & $84.4 \%$ \\
\hline Streets & $79.7 \%$ & $74.9 \%$ \\
\hline Shadows & $97.0 \%$ & $93.8 \%$ \\
\hline Bare Soil & $75.8 \%$ & $71.1 \%$ \\
\hline Total & \multicolumn{2}{|c|}{$\mathbf{8 0 . 4 2} \%$} \\
\hline
\end{tabular}

With accuracies of more than $90 \%$ - for both Landsat and TerraSAR-X classifications - the monitoring on city level gives extensive and reliable information for structural analysis. The thematic degree of detail for the high resolution satellite data enables on a high accuracy of more than $80 \%$ the analysis of urban structure on house level (Table 1). The classification of urbanised areas based on the Landsat data and the classification of sealed areas based on Quickbird data have been compared for the area of figure 5. The comparison results in $82,02 \%$ compliance. Considering that the classification result from the Quickbird fails to cover all sealed areas, the result is even more precise. But nevertheless the limited geometric potential for differentiation of urbanised from non-urbanised areas in Landsat data leads to an over classification. But still this result enables monitoring and detection of the correct dimension of spatial and temporal changes, of urban sprawl and the spatial direction of urban development. 
The results address the three questions we defined earlier in the introduction: 1) What can very different remotely sensed data sets contribute to quantify and describe structural characteristics of urban morphology and what can be reasoned from it? 2) Does spatial urbanisation correspond to the demographic development of the mega city? 3) How can urban pattern be characterised in dependency of the scale utilising remote sensing data?

1) The digital elevation model (Figure 2) shows natural limits for concentric urbanisation. The topography directs sprawl to predetermined appropriate areas. Utilising a time series of medium resolution Landsat data enables to analyse subsequent processes of the long time population pressure - as seen in figure 1 of Mexico City - which are marginal redensification in the core city as well as axial urbanisation processes along the small opening to the north (Figure 3 and 4). Most of the detected newly urbanised areas are located in the northern hinterland as well as in the southeast and show a laminar growth characteristic. In the urban core marginal and decreasing redensifcation over time is measured, while extensive urban sprawl is detected especially to the north in a distance of $20 \mathrm{~km}$ and more to the center. This is reflected in the X-and Y-Plots of the urbanised areas in figure 3. The Y-Plot shows one peak - one physical urban core -, but in recent years an indication of emerging subcenters in the north and the south-east evolves. The X-Plot reflects the spatiotemporal evolution from one physical urban core to a double-structure with two peaks caused by the axial growth lines to the north. To interpret this trend, some authors have argued that the region is showing clear signs of what Richardson (1980) called 'polarization reversal', where the secondary cities or the peripheral areas grow faster than the major city. In other words, metropolitan expansion is taking a very different form than previously, and much of the growth has shifted to a number of towns and cities within a wider metropolitan region but at some distance from the main urban center. This trend is leading to concentrated deconcentration (Aguilar, 1999).

New settlements that tend to grow in the peri-urban hinterland of cities are largely associated with poverty (de Martinez, 2007). This is because inexpensive, but mostly illegal, agricultural land has been alienated to satisfy the demands of low income population for housing. The spatiotemporal analysis of urbanised areas in combination with the DEM identifies and quantifies urban sprawl in inappropriate hillside situations. This shows that the population pressure not only leads to laminar settlements at the periphery, but also to urbanisation of hazardous areas. Figure 5 shows a detail in Mexico City located on an inappropriate area for urbanisation due to the steep slopes of the hill. The change detection utilising the multisensoral data sets (Landsat and TerraSAR-X) enables to monitor urbanisation to date. It shows that shortage of possible settlement areas in combination with population pressure leads to continuously colonisation of vulnerable areas. Areal colonisation is observed since the 1970s switching to redensification of the area from 2001 on. It shows the trend to optimised saturation of space as close as possible to urban centers or sub-centers. Thus, poverty is strongly connected to vulnerability. In this sense, risk and vulnerability in any city are socially built, and, therefore, they are not external factors to the urban process. The results can contribute to localise and quantify affected areas, assess infrastructural needs, or estimate population distribution so that a wide range of area-wide and up-to-date products supports decisionmaking.

Continuous monitoring utilising Landsat ETM data is difficult since 2003 due to technical problems of the sensor. New remotely sensed data from TerraSAR-X enable now to continue monitoring the large areas of mega cities on city level as exemplified in figure 5. The results show the capabilities of high resolution radar data to differentiate urbanised from non-urbanised areas with accuracies higher than $90 \%$. With respect to impreciseness's based on the particular data sets, both data sources proved to enable the detection of spatial urbanisation in its correct dimension. Thus, continuous change detection using multi-sensoral data sets is a promising approach for long-time studies.

2) As presented in the introduction the mega city Mexico City shows a trend of decreasing demographic growth rates starting in the 1980s but especially from the year 2000 on. In contrast, the spatial analysis of absolute areal growth for the large metropolitan area of Mexico City does not indicate decreasing land consumption from 1973 until 2001. The physical urban sprawl detected from the change detection using remotely sensed data shows an unbowed trend of urbanisation. As seen in figure 7 the urbanised areas in the metropolitan area of Mexico City almost doubled from 1973 until 2001. There is a close relation between the demographic growth of the city and its physical expansion; nevertheless, the analysis shows that the reduction of the demographic growth has been accelerated more than the physical growth 
rate. Thus, it remains to be seen if the decreasing population growth will also be reflected in decreasing land consumption in the future.

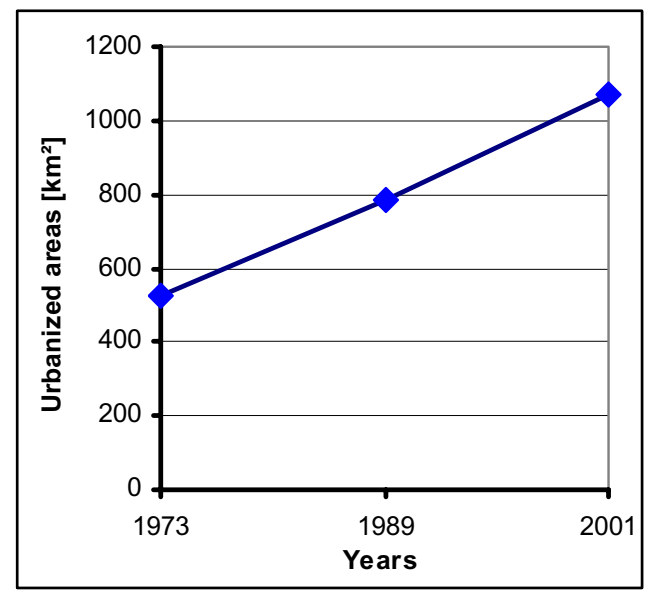

Fig. 7: Absolute areal growth in Mexico City

3) Urban structure is very much scale dependent. While the analysis on city level utilising the medium-resolution satellite data enables to assess the urban footprint and thus provides an area-wide analysis of the mega city, it does not justify the heterogeneity of its spatial pattern and structure reflected by a multitude of small-scale objects like buildings, streets and open spaces. Therefore, the multisensoral approach tries to give insight to the detailed urban structure utilising high resolution satellite data. On a higher scale the analysis of a central district reflects the typical city model of a central area in a Latin-American city. A typical chessboard street network of lower ranking back roads structures the housing blocks. The higher ranking street network does not shape the urban pattern as regulative as on housing level. On block level a consistent high built-up density of up to $80 \%$ (figure 6c) is calculated, with constant small building sizes, heterogeneous roof structures and a structured, adjacent alignment. Due to the observation that most of the time 'form follows function', these physical urban parameters reflect a typical pattern of residential housing or mixed usage. The structured and thus probably planned large buildings with lower density and large accommodation roads in the south of figure 6 indicate commercial or industrial usage. In comparison to the analysis on city level the higher degree of detail on house level enables to classify urban morphology based on a multitude on physical parameters.

\section{CONCLUSION}

The study has demonstrated that urbanisation and its spatiotemporal form, pattern and structure can be quantified utilising multi-sensoral remote sensing data. Data from optical and radar sensors proved to be an independent, area-wide, up-to-date, and adequate data source for the analysis of fast changing and large mega cities like Mexico City.

The combination of topography information with a time series of remotely sensed data as well as high resolution data sets enables to illuminate urban processes not just one-dimensional. Overall the analysis shows that urban structure is very much scale-dependent. A multi-scale analysis starting at the entire urban footprint and in addition a pattern analysis on house/block level is found to provide insight to both sides. Thus, remote sensing provides substantial multi-purpose information for describing, understanding and monitoring the spatial configuration of urban growth to support decision making in complex and multi-layered urban systems. From the need to go beyond descriptive analysis the quantification aims at reliable and comparable parameters for urban landscapes. Subsequent the attempt to correlate the results with phenomena and processes that are observed is of capital importance to understand the factors and mechanisms that 
underlie present conditions and historical trends. With this spatiotemporal information basis the lack of up-to-date data may support sustainable urban planning and management decisions.

Many observers have little confidence that such enormous conglomerations like Mexico City are manageable, many worry about the future. The synchronism and coexistence of economic activities, ecologic congestion, infrastructural deficits, poverty, population growth, etc. are huge challenges to urban planning. Therefore future research may focus on integrated interdisciplinary studies to understand the multi-dimensional and complex interactions of urban systems and to analyse effects of different measures.

\section{REFERENCES}

[1] Aguilar, A. G. (1999): Mexico City Growth and Regional Dispersal: The Expansion of the Largest Cities and New Spatial Forms. Habitat International. Vol. 23 (3). Pp. 391-412.

[2] Ávila, G.P. (2001): Urban poverty and water management. Third Annual Global Development Networks Conference. Blending local and global knowledge. Rio de Janeiro, Brazil.

[3] Batty, M. \& Howes, D. (2001): Predicting temporal patterns in urban development from remote imagery. In: J. P. Donnay, M. J. Barnsley and P.A. Longley (Hrsg.), Remote Sensing and urban analysis. S. 185-204. London. Taylor and Francis.

[4] Breunig, M. (2008): Potentiale hoch auflösender Radardaten im urbanen Raum. Unveröffentlichte Diplomarbeit. Bayerische Julius-Maximilians Universität Würzburg. (To be published).

[5] Brockerhoff, M.P. (2000): An urbanizing world. Population Bulletin. Sep 2000; 5,3; Academic Research Library pp. 3-44.

[6] Centro Nacional de Prevención de Desastres (Cenapred) (2001) : Diagnóstico de peligros e identificación de riesgos de desastres en México. Atlas nacional de riesgo de la República Mexicana, México.

[7] Martínez, A. (2007): A Study of Urban Poverty in Five Communities Living in the City of Cali (June 24, 2008). Cuadernos de Economía, Vol. 26, No. 47, 2007 Available at SSRN: http://ssrn.com/abstract=1150857.

[8] Donnay, J. P., Barnsley M. J. and Longley P. A. (2001): Remote Sensing and urban analysis. London. Taylor and Francis.

[9] DRMP (Disaster Risk Management Profile - Mexico City) (2006): 3CD City Profiles Series - Current Working Document- Mexico City, Mexico.

[10] Esch, T., Roth, A. and S. Dech (2006): Analysis of Urban Land Use Pattern Based on High Resolution Radar Imagery. Geoscience and Remote Sensing Symposium, 2006. IGARSS 2006. IEEE International Conference on Volume, Issue, July $31^{\text {th }}-$ Aug. $4^{\text {th }} 2006$, Denver. Page(s):3615 - 3618.

[11] Garza, G. (2002): New forms of urbanization: conceptualizing and measuring human settlements in the $21^{\text {st }}$ century. Paper presented at a conference on "Beyond the urban-rural dichotomy: towards a new conceptualization of human settlement systems', Bellagio, Italy, March 11-15, 2002. Mimeographed. El Colegio de Mexico, Mexico City.

[12] Gonzalez, R. R. \& Medina, J. S. (2004): Theories, Models and Urban Realities. From New York to Kathmandu. In: Dela 21, pp. 64-81.

[13] Herold, M., Goldstein, N. C., Clarke, K. C. (2003): The spatiotemporal form of urban growth: measurement, analysis and modeling. Remote Sensing of Environment 86, pp. 286-302.

[14] Herold, M., Scepan, J., Clarke, K. C. (2002): The use of remote sensing and landscape metrics to describe structures and changes in urban land uses. In: Environment and Planning A, volume 34, pp. 1443-1458.

[15] Hiernaux, N. D. (1999): Los frutos amargos de la globalización: expansión y reestructuración metropolitana de la Ciudad de México. EURE (Santiago) v.25 n.76 Santiago dic. 1999. Available at: http://www.scielo.cl/scielo.php?pid=S0250-7161999007600003\&script=sci_arttext\&tlng=es

[16] Jacquemin, A.R.A. (1999): Urban Development and New Towns in the Third World. Lessons from the New Bombay experience. Ashgate. Great Britain.

[17] Ji, W., Ma, J., Twibell, R.W and Underhill, K. (2006): Characterizing urban sprawl using mutli-stage remote sensing images and landscape metrics. In: Computers, Environment and Urban Systems, Volume 30, Issue 6, pp. 861-879. 
[18] Luck, M., Wu, J. (2002): A gradient analysis of urban landscape pattern: a case study from the Phoenixmetropolitan region, Arizona, USA. In: Landscape Ecology, vol. 17, pp. 327-339. Kluwer Academic Publishers. Richardson 1980.

[19] Mas, J.-F. (1999): Monitoring land-cover changes: a comparison of change detection techniques. In: International Journal of Remote Sensing, vol. 20, No. 1, pp. 139-152.

[20] Mertins, G. (1992): Urbanisierung, Metropolisierung und Megacities. Ursachen der Stadtexplosion in der Dritten Welt. In: Deutsche Gesellschaft für die Vereinten Nationen (Hrsg.): Mega-Städte - Zeitbombe mit globalen Folgen?, Bonn: S. 7-21.

[21] Moudon, A. V. (1997): Urban morphology as an emerging interdisciplinary field. In: Urban morphology, vol 1, pp. 3-10.

[22] O’Neill, R. V., Krummel, J. R., Gardner, R. H., Sugihara, G., Jackson, B., Deangelis, D. L., Milne, B. T., Turner, M., G., Zygmunt, B., Christensen, S. W., Dale, V. H. \& Graham, R. L. (1988): Indices of landscape pattern. In: Landscape Ecology, 1, 153-162.

[23] Pesaresi, M. (2000): Texture analysis for urban pattern recognition using fine-resolution panchromatic satellite imagery. In: Geographical and Environmental Modeling, vol. 4, no. 1, pp. 47-67.

[24] Pesaresi, M. \& Benediktson, J. A. (2001): A new approach for the morphological segmentation of high resolution satellite imagery. In: IEEE Transactions on Geoscience and Remote Sensing, vol. 39, no. 2, pp. 309-320.

[25] Pesaresi, M.\& Ehrlich, D. (2008): Spatial settlement characteristics from space. In: Gamba, P. \& Herold, M.: Global Mapping of Human Settlements: Experiences, Data Sets and Prospects. Taylor and Francis. To be published.

[26] Puissant, A., Hirsch J. \& Weber, C. (2005): The utility of texture analysis to improve per-pixel classifications for high to very high spatial resolution imagery. In: International Journal of Remote Sensing, 26(4), 733-745.

[27] Rowland A. and Gordon P. (1996): Mexico City: No longer a leviathan? In: Gilbert, A.: The Mega-City in LatinAmerica. United Nations University Press.

[28] Seto, K., C., Fragkias, M. (2005): Quantifying spatiotemporal patterns of urban land-use change in four cities of China with a time series of landscape metrics. In: Landscape Ecology 20: 871-888.

[29] Taubenböck, H. (2008): Vulnerabilitätsabschätzung der Megacity Istanbul mit Methoden der Fernerkundung. Dissertation. Universität Würzburg; p. 178. (Online-Publikation: http://www.opus-bayern.de/uniwuerzburg/volltexte/2008/2804/ ).

[30] Taubenböck, H., Wegmann, M., Berger, C., Breunig, M, Roth, A, Mehl, H. (2008): Spatiotemporal analysis of Indian mega cities. In: Proceedings of the International Archives of the Photogrammetry, Remote Sensing and Spatial Information Sciences (ISPRS). Vol. XXXVII. Part B2. Beijing 2008, pp. 75-82.

[31] Taubenböck, H., Roth, A. (2007): A transferable and stable classification approach in various urban areas and various high resolution sensors. In: Urban Remote Sensing Joint Event, Paris, France. S. 7.

[32] Taubenböck, H., Habermeyer, M., Roth, A. and Dech, S. (2006): Automated allocation of highly-structured urban areas in homogeneous zones from remote sensing data by Savitzky-Golay Filtering and curve sketching. In: IEEE Geoscience and Remote Sensing Letters. Volume 3, Issue 4, pp. 532-536. ISSN 1545-598X.

[33] Thiel, M., Esch, T., Dech, S. (2008a): Object oriented detection of settlement areas from Terra SAR-X Data. In: Carsten Jürgens (Ed.), Remote Sensing - New Challenges of High Resolution. Proceedings of the EARSeL Joint Workshop Bochum, March 5-7 2008, CD-ROM. pp 242-248.

[34] Thiel, M., Taubenböck, H., Esch, T., Roth, A., Wurm, M. \& Dech, S. (2008b): Classification of urban environments with TerraSAR-X and ALOS-PALSAR data. In: Proceedings of the SPIE - The international Society for Optical Engineering, 15-19 ${ }^{\text {th }}$ September 2008, Cardiff, Wales.

[35] United Nations (2007): World Urbanization Prospects - The 2007 revision. New York.

[36] Zhang, L., Wu, J., Zhen, Y. \& Shu, J. (2004): A GIS-based gradient analysis of urban landscape pattern of Shanghai metropolitan area, China. In: Landscape Urban Plan. 69, 1-16. 